\title{
An analysis of the perceived causes leading to task-failure in resistance-exercises
}

\author{
Aviv Emanuel ${ }^{1,2,3}$, Isaac Isur Rozen Smukas ${ }^{2,3}$, Israel Halperin ${ }^{\text {Corresp. 2, } 3}$ \\ ${ }^{1}$ School of Psychological Sciences, Tel Aviv University, Tel Aviv, Israel \\ ${ }^{2}$ School of Public Health, Sackler Faculty of Medicine, Tel Aviv University, Tel Aviv, Israel \\ 3 Sylvan Adams Sports Institute, Tel Aviv University, Tel Aviv, Israel \\ Corresponding Author: Israel Halperin \\ Email address: ihalperin@tauex.tau.ac.il
}

Background: While reaching task-failure in resistance-exercises is a topic that attracts scientific and applied interest, the underlying perceived reasons leading to task-failure remain underexplored. Here, we examined the reasons subjects attribute to task-failure as they performed resistance-exercises using different loads. Methods: Twenty-two resistance-trained subjects (11-females) completed one Repetition-Maximum (RM) tests in the barbell squat and bench-press. Then, in the next two counterbalanced sessions, subjects performed two sets to task-failure in both exercises, using either $70 \%$ or $83 \%$ of 1RM. Approximately 30 seconds after set-completion, subjects verbally reported the reasons they perceived to have caused them to reach task-failure. Their answers were recorded, transcribed, and thematically analyzed. The differences between the frequencies of the identified categories were then tested using a mixed logistic regression model. Results: The most commonly reported reason was muscle fatigue ( $54 \%, p<0.001)$, mostly of the target muscles involved in each exercise. However, remote muscles involved to a lesser extent in each exercise were also reported. Approximately half of the remaining reasons included general fatigue (26\%), pain (12\%), cardiovascular strain (11\%), and negative affect $(10 \%)$, with the latter three reported more often in the squat $(p=0.022)$. Conclusions: In contrast to our expectations, task-failure was perceived to be caused by a range of limiting factors other than fatigue of the target muscles. It now remains to be establishedwhether different perceived limiting factors of resistance-exercises lead to different adaptations, such as muscular strength and hypertrophy. 


\section{An analysis of the perceived causes leading to task-failure in}

\section{2 resistance-exercises}

3 Aviv Emanuel ${ }^{1,2,3}$ Isaac Isur Rozen Smukas ${ }^{2,3}$ and Israel Halperin ${ }^{2,3 *}$

4

$5 \quad{ }^{1}$ School of Psychological Sciences, Tel-Aviv University, Tel-Aviv, Israel

$6 \quad{ }^{2}$ School of Public Health, Sackler Faculty of Medicine, Tel-Aviv University, Tel-Aviv, Israel

$7 \quad$ SSylvan Adams Sports Institute, Tel Aviv University, Tel-Aviv, Israel

8

9 Corresponding author:

10 Israel Halperin ${ }^{2,3}, 55$ Haim Levanon st. Tel-Aviv, 69978 Israel.

11 Email address: ihalperin@tauex.tau.ac.il 
19 Abstract

20 Background: While reaching task-failure in resistance-exercises is a topic that attracts scientific

21 and applied interest, the underlying perceived reasons leading to task-failure remain

22 underexplored. Here, we examined the reasons subjects attribute to task-failure as they

23 performed resistance-exercises using different loads.

24 Methods: Twenty-two resistance-trained subjects (11-females) completed one Repetition-

Maximum (RM) tests in the barbell squat and bench-press. Then, in the next two counterbalanced sessions, subjects performed two sets to task-failure in both exercises, using either $70 \%$ or $83 \%$ of $1 \mathrm{RM}$. Approximately 30 seconds after set-completion, subjects verbally reported the reasons they perceived to have caused them to reach task-failure. Their answers were recorded, transcribed, and thematically analyzed. The differences between the frequencies of the identified categories were then tested using a mixed logistic regression model.

Results: The most commonly reported reason was muscle fatigue $(54 \%, p<0.001)$, mostly of the target muscles involved in each exercise. However, remote muscles involved to a lesser extent in each exercise were also reported. Approximately half of the remaining reasons included general fatigue $(26 \%)$, pain $(12 \%)$, cardiovascular strain $(11 \%)$, and negative affect $(10 \%)$, with the latter three reported more often in the squat $(p=0.022)$.

Conclusions: In contrast to our expectations, task-failure was perceived to be caused by a range of limiting factors other than fatigue of the target muscles. It now remains to be established whether different perceived limiting factors of resistance-exercises lead to different adaptations, such as muscular strength and hypertrophy.

Keywords: Resistance-exercise, perception, task-failure, repetition-maximum 


\section{Introduction}

42 Whether one should reach task-failure in resistance-exercises is a question that attracts scientific and applied interest (Nóbrega \& Libardi, 2016; Davies et al., 2016; Sampson \& Groeller, 2016). Here we refer to task-failure as either momentary-failure (MF), the point in which an attempted repetition cannot be completed with proper form, or repetition maximum (RM), the final repetition one can complete prior to reaching MF (Steele et al., 2017). It has been established that reaching or approaching task-failure is important for hypertrophy and strength development (Davies et al., 2016; Morton et al., 2016). Yet, to date, little is known about the subjective reasons underpinning task-failure. Although it can be expected that the inability to generate the required force with the target muscles is the main reason for task-failure, perception of fatigue, negative feelings, cardiovascular strain and pain can also be at play. Reaching task-failure due to one or more of the aforementioned factors could lead to different adaptations. For example, reaching task-failure because one cannot generate enough force with the target muscles is possibly more effective for hypertrophy purposes compared to reaching task-failure because of cardiovascular strain, or pain in body parts other than the target muscle groups. While investigating the underlying physiological causes of task-failure is a challenging task, examining the subjective aspects believed to cause task-failure is attainable and can shed light on this important issue.

One effective way to study subjective experiences during resistance training is with single-item scales (Buckley \& Borg, 2011; Helms et al., 2016; Hackett et al., 2017). For example, rating of perceived effort (RPE) scales, such as Borg CR-10 (e.g., Buckley \& Borg, 2011), can assist quantifying how much effort one is investing during, or after set completion, and allow for comparisons between different exercises, loads, and body parts involved in the exercise (Duncan, 
64 Al-Nakeeb \& Scurr, 2006; Buckley \& Borg, 2011). Interestingly, when sets are taken to taskfailure, perceived effort is not always rated as maximal, indicating that effort is not the limiting factor in such cases (Pritchett et al., 2009). Such results led investigators to examine if other constructs can be the reason people terminate sets (Steele et al., 2016). A number of studies found that perception of discomfort, rather than RPE, was maximal at the point of settermination, suggesting that discomfort can be a limiting factor (Fisher \& Steele, 2017; Stuart et al., 2018).

Other popular effort scales gauge how many repetitions trainees estimate they can complete before reaching task-failure (Helms et al., 2016; Hackett et al., 2017). To illustrate, a rating of two indicates that only two more repetitions are left before reaching task-failure (Hackett et al., 2017). These scales are similar to RPE scales although developed solely for resistance training (Helms et al., 2016; Hackett et al., 2017). While single-item scales are practically useful and lead to theoretical insights (Halperin \& Emanuel, 2019), they lack the resolution required to pinpoint the reasons why task-failure occurs. This is because they depend on a single question, which revolves around a single construct, to which people can only provide a single answer. Hence, other strategies of investigations are warranted.

Another way to investigate the causes leading to task-failure is to ask subjects why they terminated a set, and allow them to answer in an unrestrictive manner. This measurement strategy can expand our understanding of set termination that goes beyond the insightful, yet limited knowledge gathered via single-item scales. Such knowledge could lead to new research avenues. The limiting factors may vary as a function of the exercises completed and the loads lifted. Hence, the purpose of the present work was to examine the perceived causes trainees attribute to task-failure across different loads and exercises. To achieve this goal, we instructed 
87 resistance-trained subjects to reach task-failure in the squat and bench-press exercises under two

88 load conditions (70\% and $83 \%$ of $1 \mathrm{RM})$. Within $20-40$ seconds after set completion, we asked

89 them what was the limiting factor in the set, and why they did not perform another repetition.

90 Subjects' answers were recorded, transcribed and analyzed.

91 Materials \& Methods

92 Study design

93 The study consisted of three testing days with three to eight days between sessions. The first day

94 consisted of 1RM tests for the barbell back-squat followed by the barbell bench-press, and an

95 explanation of the experimental procedure. In the following two sessions, subjects performed

96 two sets with the barbell squat followed by two sets with the barbell bench-press to task-failure,

97 while lifting either $70 \% 1 \mathrm{RM}$ in one session or $83 \% 1 \mathrm{RM}$ in the other, in a randomized,

98 counterbalanced order. While subjects were asked to reach MF in all sets, they were informed

99 that they can terminate the set at RM. Hence, across days, task-failure was determined either by

100 1) inability to complete a repetition (MF), 2) subjects' decision to terminate the set based on their

101 assumption that they can’t complete another repetition (RM), or 3) technical failure determined

102 by the experimenter.

103 The $70 \%$ and $83 \%$ of $1 \mathrm{RM}$ loads were selected for two reasons. First, they are within the

104 recommended range for development of hypertrophy and strength for trainees with a range of

105 training backgrounds (Kraemer \& Ratamess, 2004). Second, based on pilot work and a previous

106 study from our lab (Emanuel, Rozen Smukas \& Halperin, 2020a), the two loads were expected to

107 lead to considerable differences in the number of repetitions subject complete, yet lifting loads

108 heavier than $83 \% 1 \mathrm{RM}$ could have led subjects to perform as little as two or three repetitions in 
109 the final sets - an outcome we were preferred to avoid. Note that this this study is based on the

110 protocol used in Emanuel et al. (2020b) but addressed outcomes which were previously

111 unexamined.

112 In the two experimental sessions, after each set, the researcher noted if the set was terminated

113 due to RM or MF. The researcher then asked the subjects what were the limiting factors in the 114 set, and why they could not perform another repetition. Subjects answered this question as they

115 saw fit, without any restrictions. All answers were recorded with a tie-on microphone attached to

116 the subjects' shirt and were later transcribed and analyzed. Subjects were asked to refrain from

117 an intense training session 24 hours prior to testing days and to avoid a heavy meal and

118 caffeinated drinks or supplements at least three hours before testing sessions. All sessions were

119 performed in the same facilities and ran by the same experimenter at approximately the same

120 hour of the day ( \pm 2 hours). No individuals other than the same single experimenter and a single

121 subject were allowed to enter the lab during the experimental sessions.

\section{Subjects}

123 Twenty-two resistance trained subjects volunteered to participate in this study (Table 1). Due to

124 the exploratory nature of this work, a power analysis was not conducted. Yet, we assumed that

125 subjects would perform at least 150 sets, which would provide us with an adequate amount to

126 data to explore the topic at hand. Inclusion criteria consisted of healthy subjects between the ages

127 of 18 and 45; a bench-press 1RM of at least 1.2 and 0.7 times the bodyweight for men and

128 women, respectively; and at least 1.2, and 1 times the bodyweight in the squat. Subjects had to

129 have at least one year of resistance training experience, and specifically at performing the free

130 weight squat and bench-press. Additionally, subjects had to have some familiarity with taking 
131 sets to task-failure. Each subject signed an informed consent on the first day. This study was

132 approved by the Tel-Aviv University institutional review 2019-0325.

\section{Procedures}

134 1RM tests (day 1). Subjects were first weighed (Xinfu Household Electronics, Guandong,

135 China) indicated their height, age and experience in strength training. They were then briefed on 136 the study's aims, namely, to measure different perceptions associated with performance of the

137 bench press and squat exercises to task-failure, across two different loads. All subjects then 138 performed a squat to a height adjustable box which was set to achieve a knee angle of 115-120 degrees measured with a goniometer (mean knee angle $=118, \mathrm{SD}=5.93$ ). Subjects had to lightly touch the box with their gluteus prior to initiating the concentric phase. During the bench-press subjects' preferred grip and body position were recorded and maintained throughout the study. In each repetition the bar must have lightly touched subjects' chest prior to the concentric phase.

Subjects then performed a structured warmup protocol consisting of calisthenics and dynamic warmup followed by an individualized five-minutes warmup. This warmup protocol was identical in all sessions. Subjects then performed the barbell squat and bench-press 1RM protocol which consisted of a similar progression towards an estimated 1RM indicated by the subjects: $10,5,3,3,2,1$ repetitions with empty bar, $40 \%, 60 \%, 70 \%, 80 \%$ and $90 \%$ of approximated $1 \mathrm{RM}$, respectively. The increase in weight to the true 1RM was decided by the subjects and experimenter with 3-5 minutes of rest between 1RM attempts.

Experimental sessions (day 2-3). Following the general warmup protocol (see above), subjects

151 performed the following specific warmup for the squat and again for the bench-press following 152 the sets to task-failure with the squat: $10,5,3,1$ repetitions with an empty bar with $40 \%, 55 \%$, $15370 \%$ of $1 \mathrm{RM}$ in the lighter day, or added another set of one repetition with $83 \%$ of $1 \mathrm{RM}$ in the 
154 heavier day. Following the last warmup set, subjects rested for two minutes and performed two

155 sets to task-failure in the squat followed by the bench press with either $70 \%$ or $83 \%$ of 1 RM. Six

156 minutes of rest were provided between sets and exercises. Subjects were instructed to perform

157 the concentric portion of the lift as fast as possible, while maintaining a controlled $\sim 2$ seconds

158 descend, as was assessed and insured by the experimenter. Within 20-40 seconds after set

159 completion, subjects were asked what were the limiting factors in the set, and why they could not

160 perform another repetition. The 20-40 seconds wait ensured subjects could catch their breath and

161 sit down before providing an answer.

162 (Table 1 near here)

163 Data preparation

164 We followed a similar approach used by Halperin et al. (2016). Initially, general categories that

165 were expected to account for set termination were extensively discussed by the authors. These

166 categories included general and specific muscle fatigue, pain, negative affect, and cardiovascular

167 strain (see below). Then, the first and last authors read all of the transcribed statements and

168 examined if the agreed upon categories were present, needed to be refined, and whether other

169 ones were noticed. In case that newer categories were identified, a discussion took place in order

170 to decide if they should be included. Once the categories were agreed upon, the first and last

171 authors picked at random a few statements and rated them simultaneously to confirm comparable

172 ratings. Thereafter, the raters rated all statements individually. Each statement was rated once in

173 a binary manner in each category. The raters then compared their overall ratings. Cases of

174 mismatches were thoroughly discussed between the two raters until reaching an agreement. Note

175 that the same statement could have been rated in more than one category. The final categories

176 included the following:

Peer) reviewing PDF | (2020:04:48339:1:1:CHECK 13 Jun 2020) 
177 General fatigue: statements with terms such as fatigue, tired, lack of energy, power or strength,

178 all in relation to the whole body, or described as a general perception.

179 Specific fatigue: as described above, but the perceptions had to be attributed to muscles or a 180 location in the body.

181 Cardiovascular strain: statements indicating that breathlessness or heartrate were the reasons for 182 set-termination.

183 Pain: statements indicating a painful experience, including terms such as pain, hurt, pinch, and 184 burning.

185 Negative affect: statements indicating an overall bad experience including terms such as 186 annoyance, bad, terrible, and not fun.

187 Table 2 provides examples of the statements provided in each of the five categories across 188 exercises, loads, RM/MF and gender, and their rating in each category. Since subjects provided 189 the answers in Hebrew, the first author, who is fluent in both Hebrew and English, translated the 190 statements into English.

191 (Table 2 near here)

\section{Data analysis}

193 We tested for differences between the frequencies of the categories across loads and exercises in 194 a mixed logistic regression model (the cardiovascular category was coded as 0 ). We next tested 195 for differences in the frequency of each category between conditions and exercises via five 196 separate mixed logistic regression models, one for each category (the frequency in a given 197 category was the dependent variable while condition and exercise were the independent 
198 variables). In all mixed regression models, random effects and interactions were added based on 199 improvement in model fit, as indicated by the deviance statistic. In addition, the specific body 200 parts mentioned, if any, under the muscle fatigue and pain categories were mapped (e.g., upper-

201 body, quadriceps), counted, and reported. We also counted the frequencies of each category 202 separately for males and females for exploratory purposes. Significance was set at $p<0.05$. 203 Statistical analyses and figures were carried out in R (version 3.6.0, R Core Team, Vienna, 204 Austria) using the lme4 (Bates et al., 2015), cowplot (Wilke, 2017), and ggplot2 (Wickham, 205 2011) packages.

\section{Results}

207

208

209

210

211

212

213

214

215

216

217

218

219

Overall, 158 statements from terminated sets were recorded with only 37 sets terminated due to MF and the rest due to RM. We had ten missing observations in the $83 \% 1 \mathrm{RM}$ bench-press condition; four due to drop-outs and six due to technical difficulties. We had four more missing observations across the other conditions and exercises due to technical difficulties. The mean number of repetitions completed across sets and gender in the 70\%1RM condition was $16.4 \pm 8.9$ (range: 6-55) and 13 \pm 2.9 (range: 7-19) in the squat and bench-press respectively, whereas in the $83 \% 1 \mathrm{RM}$ it was $8.1 \pm 3.3$ (range: $3-17$ ) and $7.1 \pm 1.8$ (range: $4-12$ ) in the squat and bench-press respectively.

Overall frequencies of each category. The final mixed logistic regression model included a random intercept, and its fixed effects were defined by the following equation:

$$
\text { Frequency } y_{i j} \sim \operatorname{logistic}\left(\text { Category }_{i j}+u_{0 j}\right)
$$

Out of the 158 statements, muscle fatigue was found to be the most frequent category accounting for $53.8 \%$ of the terminated sets $(O R=9, b=2.2, S E=0.29, z=7.42, p<0.001,95 \% \mathrm{CI}[1.8,2.9])$. The 
220 second most frequent category was general fatigue accounting for $25.9 \%$ of the terminated sets

$221(O R=2.7, b=1, S E=0.31, z=3.24, p=0.001,95 \% \mathrm{CI}[0.45,1.67])$, followed by pain $(12 \%)$,

222 cardiovascular (11.4\%) and negative affect (10.1\%). No significant differences were found

223 between the frequency of either pain $(O R=1, b=0.06, S E=0.35, z=0.17, p=0.861,95 \% \mathrm{CI}[-0.66$,

$2240.74])$ or negative affect $(O R=0.87, b=-0.13, S E=0.36, z=-0.36, p=0.716,95 \% \mathrm{CI}[-0.87,0.59])$

225 and the cardiovascular category (See Figure 1).

226 Differences in frequencies between exercises and loads, males and females. The results of the

227 mixed logistic regression models tested for each separate category are presented at Table 3 . The

228 frequencies the categories by gender are reported in Table 4.

229 (Figure 1 near here)

230 (Table 3 near here)

231 (Table 4 near here)

\section{Discussion}

233 Here we examined what limiting factors were perceived to lead to task-failure in the squat and

234 bench-press exercises using $70 \%$ and $83 \%$ of 1 RM, among resistance-trained subjects. Subjects

235 were required to state what were the limiting factors of each set within 20-40 seconds after

236 reaching task-failure. Of the five categories used to map subjects' responses, specific muscle

237 fatigue was the most frequently reported. Within the muscle fatigue category, the target muscles

238 (i.e., prime movers) in each exercise were specified in most, but not all of the responses. The

239 remaining responses were categorized as general fatigue, pain, negative affect and cardiovascular

240 strain. Some differences were observed between exercises with task-failure in the squat being

241 attributed more often to negative affect and, while statistically insignificant, in cardiovascular 
242 strain compared to the bench-press exercise. Similarity, some differences were observed between

243 loads, in which lighter loads led to greater pain and cardiovascular strain compared to heavier

244 loads. No differences in the distribution of categories were identified between males and

245 females.

246 As can be expected, most sets were perceived to be terminated because of muscle fatigue

247 attributed to target muscle groups (i.e., lower body for squats and upper body for bench-press).

248 However, within this category, certain variability was noted. In the squat, 27\% (13/47) of the

249 statements within the muscle fatigue category were attributed to the upper body and lower back,

250 indicating that in these cases, task-failure was not perceived to be due to the target muscles.

251 Moreover, different muscles within the legs were mentioned, including the gluteus, quadriceps

252 and hamstring, indicating that limiting factors within the target muscle groups can vary between

253 individuals. In the bench-press, some variability was also noted within the target muscle groups,

254 with the chest muscles being reported the most, followed by the arms (presumed to be triceps)

255 and then the shoulders. The variability in reporting which of the target muscle groups was the

256 limiting factor in each exercise can partly be explained by technical variation in the way the

257 exercises were performed. For example, different stance widths, foot placement, and forward

258 lean during the squat can affect the moments at the hip and knee joints (Escamilla et al., 2001;

259 Glassbrook et al., 2017; Lorenzetti et al., 2018). It is interesting to consider whether variations in

260 the perceived muscle group being the limiting factor in a given exercise can lead to different

261 adaptations, such as strength and hypertrophy. Alternatively, whether suboptimal adaptations

262 may occur when unrelated muscles are considered to be the limiting factor in exercise-

263 performance. 
264 General fatigue was also frequently attributed to set termination across exercise and loads. This

265 could indicate that multi-joint exercises involving large muscle groups produce a global and

266 unspecified feeling of fatigue strong enough to lead to set-termination. While pain, negative

267 affect, and cardiovascular strain were mentioned fewer times compared to specific and general

268 fatigue, some interesting patterns emerged. Mainly, the results indicate that more sets in the

269 squats were terminated due to pain and negative affect compared to bench-presses, and that in

270 the $70 \% 1 \mathrm{RM}$ squat condition more sets were terminated due to cardiovascular strain. Some of

271 these findings are aligned with other studies. For example, lower body exercises have shown to

272 cause greater degree of negative affect (Portugal et al., 2015), and require greater energy

273 expenditure (Lyons et al., 2007; Flanagan et al., 2014; Vianna et al., 2014) compared to upper

274 body exercises. These findings suggest that squats may be limited by a wider range of factors,

275 compared to the bench-press. As a whole, these results are also alighted with other studies

276 reporting that other constructs, such as discomfort, can be a limiting factor during sets (Steele et

277 al., 2016).

278 This study has several methodological aspects worthy of discussion. First, asking subjects to

279 answer the question concerning set termination within $\sim 30$ seconds can be viewed both as a

280 strength and a limitation. Asking the question in proximity to set termination was expected to

281 lead to a more accurate answer, but given the physically challenging nature of the task, subjects

282 may have provided less details. Similarly, subjects were allowed to answer the question without

283 any restrictions, but this may have caused important information to be lost that a more structured

284 questioning procedures might have captured. Second, only $23 \%$ of sets were completed due to

$285 \mathrm{TF}$, despite subjects being encouraged to reach TF in all sets. It is possible that set termination

286 due to RM and TF lead to different perceptions of its underlying causes. However, since it is 
287 ethically impossible to enforce $\mathrm{TF}$, overcoming this limitation is a challenging task. Third, while

288 subjects in this study were experienced in RT, their unique training background could have

289 shaped their responses. Moreover, subjects' beliefs and attitudes about the factors leading to set

290 termination, as well as their general knowledge pertaining to resistance training may have also

291 played a role in their responses (e.g., Vaegter et al., 2020). Third, no physiological outcome,

292 such as heartrate, was measured and correlated with participants' statements. In view of the

293 aforementioned points, future studies should collect more data on trainees' training background

294 and knowledge, attitudes and perception using structured or semi-structured interviews and also

295 collect physiological measures.

296

297

298

299

300

301

302

303

304

305

306

307

308

309

As studies continue to investigate how many repetitions one completes relative to task-failure, it may be of added value to examine what are the limiting factors leading to set-termination. This is because different limiting factors within sets could lead to different acute and long-term adaptations that might not be aligned with the sought-after outcomes. Investigating limiting factors can be done by directly measuring the possible physiological pathways leading to settermination. Such studies require complex designs and equipment. An alternative is to study the subjective experiences accounting for set-termination, as was done in the present study, and to our knowledge, for the first time. The present investigation may indicate that relying on taskfailure as a standard for load and repetition prescription may fail to capture a variety of limiting factors other than fatigue of the target muscles. Thus, it could be that in addition to prescribing one to reach task-failure, or proximity to task-failure, monitoring other aspects of set-termination may prove beneficial. For example, loads can be modified in cases that cardiovascular strain or negative affect are reported at set-termination, and exercise technique can be modified in cases that target muscles are not perceived to be a limiting factor. However, it is required to first 
310 establish what type of relationships, if any, exist between perception of the limiting factors and

311 actual adaptations.

\section{Conclusions}

313 We observed that set-termination was mostly perceived by subjects to be a result of muscle

314 fatigue in the target muscles, followed by a general feeling of fatigue, negative affect, pain and

315 cardiovascular causes. These reasons were found to vary between exercises and loads. The

316 results of this study show that there are a variety of perceived reasons for set termination, which

317 might affect exercise-adaptations, and warrant further investigation.

\section{References}

319

320

321

322

Bates D, Maechler M, Bolker B, Walker S, Christensen RHB, Singmann H, Dai B, Grothendieck G, Green P, Bolker MB. 2015. Package 'Ime4.' Convergence 12:2.

Buckley JP, Borg GAV. 2011. Borg's scales in strength training; from theory to practice in young and older adults. Applied Physiology, Nutrition, and Metabolism 36:682-692. DOI: $10.1139 / \mathrm{h} 11-078$.

Davies T, Orr R, Halaki M, Hackett D. 2016. Effect of Training Leading to Repetition Failure on Muscular Strength: A Systematic Review and Meta-Analysis. Sports Medicine 46:487502. DOI: 10.1007/s40279-015-0451-3.

Duncan MJ, Al-Nakeeb Y, Scurr J. 2006. Perceived exertion is related to muscle activity during leg extension exercise. Research in Sports Medicine 14:179-189.

Emanuel, A., Rozen Smukas, I. Halperin, I., in press. How one feels during resistance exercises: A repetition by repetition analysis across exercises and loads. International Journal of Sports Physiology and Performance. 
332 Emanuel, A., Rozen Smukas, I. Halperin, I., in press. The effects of lifting lighter and heavier

333

334

335

336

337

338

339

340

341

342

343

344

345

346

347

348

349

350

351

352

353 loads on subjective measures. International Journal of Sports Physiology and Performance.

Escamilla RF, Fleisig GS, Zheng N, Lander JE, Barrentine SW, Andrews JR, Bergemann BW, Moorman III CT. 2001. Effects of technique variations on knee biomechanics during the squat and leg press. Medicine \& Science in Sports \& Exercise 33:1552-1566.

Fisher JP, Steele J. 2017. Heavier and lighter load resistance training to momentary failure produce similar increases in strength with differing degrees of discomfort: Responses to Training Loads. Muscle \& Nerve 56:797-803. DOI: 10.1002/mus.25537.

Flanagan SD, Mills MD, Sterczala AJ, Mala J, Comstock BA, Szivak TK, DuPont WH, Looney DP, McDermott DM, Hooper DR. 2014. The relationship between muscle action and repetition maximum on the squat and bench press in men and women. The Journal of Strength \& Conditioning Research 28:2437-2442.

Glassbrook DJ, Helms ER, Brown SR, Storey AG. 2017. A review of the biomechanical differences between the high-bar and low-bar back-squat. The Journal of Strength \& Conditioning Research 31:2618-2634.

Hackett DA, Cobley SP, Davies TB, Michael SW, Halaki M. 2017. Accuracy in Estimating Repetitions to Failure During Resistance Exercise: Journal of Strength and Conditioning Research 31:2162-2168. DOI: 10.1519/JSC.0000000000001683.

Halperin I, Chapman DW, Martin DT, Abbiss C, Wulf G. 2016. Coaching cues in amateur boxing: An analysis of ringside feedback provided between rounds of competition. Psychology of Sport and Exercise 25:44-50. DOI: 10.1016/j.psychsport.2016.04.003.

Peer) reviewing PDF | (2020:04:48339:1:1:CHECK 13 Jun 2020) 
354 Halperin I, Emanuel A. 2019. Rating of Perceived Effort: Methodological Concerns and Future 355 Directions. Sports Medicine. DOI: 10.1007/s40279-019-01229-z.

356 Helms ER, Cronin J, Storey A, Zourdos MC. 2016. Application of the Repetitions in Reserve-

357 Based Rating of Perceived Exertion Scale for Resistance Training: Strength and Conditioning Journal 38:42-49. DOI: 10.1519/SSC.0000000000000218.

359

360

361

362

363

364

365

366

367

368

369

370

371

372

373

374

375

Kraemer WJ, Ratamess NA. 2004. Fundamentals of Resistance Training: Progression and Exercise Prescription: Medicine \& Science in Sports \& Exercise 36:674-688. DOI: 10.1249/01.MSS.0000121945.36635.61.

Lorenzetti S, Ostermann M, Zeidler F, Zimmer P, Jentsch L, List R, Taylor WR, Schellenberg F. 2018. How to squat? Effects of various stance widths, foot placement angles and level of experience on knee, hip and trunk motion and loading. BMC Sports Science, Medicine and Rehabilitation 10:14.

Lyons S, Richardson M, Bishop P, Smith J, Heath H, Giesen J. 2007. Excess post-exercise oxygen consumption in untrained men following exercise of equal energy expenditure: comparisons of upper and lower body exercise. Diabetes, Obesity and Metabolism 9:889-894.

Morton RW, Oikawa SY, Wavell CG, Mazara N, McGlory C, Quadrilatero J, Baechler BL, Baker SK, Phillips SM. 2016. Neither load nor systemic hormones determine resistance training-mediated hypertrophy or strength gains in resistance-trained young men. Journal of Applied Physiology 121:129-138. DOI: 10.1152/japplphysiol.00154.2016.

Nóbrega SR, Libardi CA. 2016. Is Resistance Training to Muscular Failure Necessary? Frontiers in Physiology 7. DOI: 10.3389/fphys.2016.00010. 
376 Portugal EMM, Lattari E, Santos TM, Deslandes AC. 2015. Affective Responses to Prescribed

377

378

379

380

381

382

383

384

385

386

387

388

389

390

391

392

393

394

395

396

397 and Self-Selected Strength Training Intensities. Perceptual and Motor Skills 121:465481. DOI: $10.2466 / 29 . P M S .121 \mathrm{c} 17 \times 3$.

Pritchett R, Green J, Wickwire P, Kovacs M. 2009. Acute and session RPE responses during resistance training: Bouts to failure at $60 \%$ and $90 \%$ of 1RM. South African Journal of Sports Medicine 21. DOI: 10.17159/2078-516X/2009/v21i1a304.

Sampson JA, Groeller H. 2016. Is repetition failure critical for the development of muscle hypertrophy and strength?: Failure is not necessary for strength gain. Scandinavian Journal of Medicine \& Science in Sports 26:375-383. DOI: 10.1111/sms.12445.

Steele J, Fisher J, Giessing J, Gentil P. 2017. Clarity in reporting terminology and definitions of set endpoints in resistance training: Issues \& Opinions: Resistance Training Endpoints. Muscle \& Nerve 56:368-374. DOI: 10.1002/mus.25557.

Steele J, Fisher J, McKinnon S, McKinnon P. 2016. Differentiation between perceived effort and discomfort during resistance training in older adults:Reliability of trainee ratings of effort and discomfort,and reliability and validity of trainer ratings of trainee effort. Journal of Trainology 6:1-8. DOI: 10.17338/trainology.6.1_1.

Stuart C, Steele J, Gentil P, Giessing J, Fisher JP. 2018. Fatigue and perceptual responses of heavier- and lighter-load isolated lumbar extension resistance exercise in males and females. PeerJ 6:e4523. DOI: 10.7717/peerj.4523.

Vaegter HB, Thinggaard P, Madsen CH, Hasenbring M, Thorlund JB. 2020. Power of Words: Influence of Preexercise Information on Hypoalgesia after Exercise-Randomized Controlled Trial. Medicine and Science in Sports and Exercise. 
398 Vianna JM, Werneck FZ, Coelho EF, Damasceno VO, Reis VM. 2014. Oxygen uptake and heart 399 rate kinetics after different types of resistance exercise. Journal of Human Kinetics $400 \quad 42: 235-244$.

401 Wickham H. 2011. ggplot2. Wiley Interdisciplinary Reviews: Computational Statistics 3:180$402 \quad 185$.

403 Wilke CO. 2017. cowplot: Streamlined Plot Theme and Plot Annotations for "ggplot2”. R $404 \quad$ package version 0.9. 4.

\section{Figure captions}

406 Figure 1. The distribution of each category by exercises and loads. Panels A-E depict the muscle 407 fatigue, general fatigue, pain, cardiovascular, and negative affect categories, respectively. The y408 axis represents the percent of each category, calculated as the number of ratings, divided by the 409 number of sets in each exercise and load. 


\section{Figure 1}

The distribution of each category by exercises and loads.

(A-E) depict the muscle fatigue, general fatigue, pain, cardiovascular, and negative affect categories, respectively. The $y$-axis represents the percent of each category, calculated as the number of ratings, divided by the number of sets in each exercise and load.
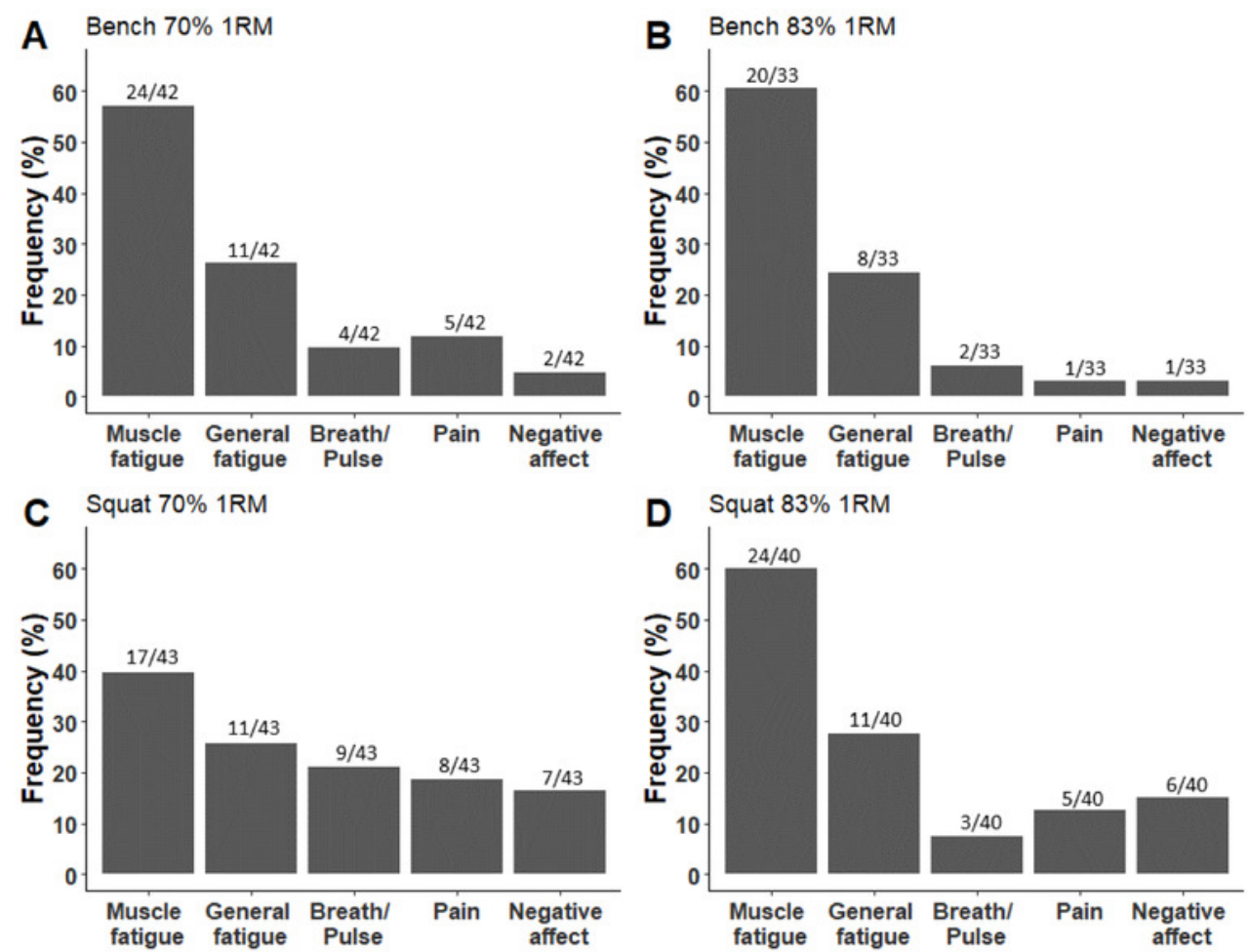
Table $\mathbf{1}$ (on next page)

Table 1. General Demographics

General Demographics. 
Table 1. General Demographics.

\begin{tabular}{lcc}
\hline & $\begin{array}{c}\text { Females } \\
(\mathrm{n}=11) \\
\text { Mean } \pm \text { SD } \\
(\text { Range) }\end{array}$ & $\begin{array}{c}\text { Males } \\
(\mathrm{n}=11) \\
\text { Mean } \pm \text { SD } \\
(\text { Range })\end{array}$ \\
\hline Age & $29 \pm 4$ & $30 \pm 4$ \\
& $(23-38)$ & $(22-37)$ \\
\hline Height (cm) & $167 \pm 6$ & $175 \pm 6$ \\
& $(156-175)$ & $(167-185)$ \\
\hline Weight (kg) & $62 \pm 7$ & $78 \pm 4$ \\
& $(52-75)$ & $(71.7-85.7)$ \\
\hline Experience in RT (yrs) & $3 \pm 2$ & $9 \pm 4$ \\
& $(1-8)$ & $(3-18)$ \\
\hline Mean workouts per week & $3 \pm 1$ & $3.5 \pm .8$ \\
& $(1-5)$ & $(2-5)$ \\
\hline 1RM barbell bench press (kg) & $45 \pm 9$ & $100 \pm 14$ \\
& $(31-60)$ & $(75-130)$ \\
\hline 1RM/ Bodyweight bench press & $0.71 \pm 0.12$ & $1.29 \pm 0.22$ \\
& $(0.53-0.87)$ & $(1-1.7)$ \\
\hline 1RM barbell squat (kg) & $74 \pm 14$ & $126 \pm 20$ \\
& $(55-100)$ & $(100-155)$ \\
\hline 1RM/ Bodyweight squat & $1.19 \pm 0.24$ & $1.6 \pm 0.27$ \\
& $(0.81-1.50)$ & $(1.2-1.6)$ \\
\hline & & \\
& & \\
& &
\end{tabular}




\section{Table 2 (on next page)}

\section{Table 2. Example of statements}

Examples of answers provided to the question posed after set-completion: "What were the limiting factors of the set and why couldn't you perform another repetition?". The ratings of each answer are divided by category, exercise, load, and set endpoint condition $(*$ indicates MF). 
Table 2. Examples of answers provided to the question posed after set-completion: "What were the limiting factors of the set and why couldn't you perform another repetition?". The ratings of each answer are divided by category, exercise, load, and set endpoint condition (* indicates MF).

\begin{tabular}{|c|c|c|c|c|c|c|}
\hline Load & Answers & $\begin{array}{l}\text { Muscle } \\
\text { Fatigue }\end{array}$ & $\begin{array}{l}\text { General } \\
\text { Fatigue }\end{array}$ & Affect & Cardio & Pain \\
\hline \multicolumn{7}{|c|}{ Squats } \\
\hline $70 \%$ & A general feeling of exhaustion. & & $\mathbf{x}$ & & & \\
\hline $70 \% *$ & My quadriceps and hamstring muscles. & $\mathbf{x}$ & & & & \\
\hline $70 \%$ & $\begin{array}{l}\text { My lower back and my left quadriceps but also an unpleasant } \\
\text { feeling in my body. }\end{array}$ & $\mathbf{x}$ & & $\mathbf{x}$ & & \\
\hline $70 \%$ & I didn't reach failure, I just had enough. & & & $\mathbf{x}$ & & \\
\hline $83 \%$ & $\begin{array}{l}\text { I couldn't push with my legs any more, } \\
\text { I feel fatigued. }\end{array}$ & $\mathbf{x}$ & $\mathbf{x}$ & & & \\
\hline $83 \%$ & I felt stuck and a pressure in my lower back muscles. & & $\mathbf{x}$ & & & $\mathbf{x}$ \\
\hline $83 \%$ & $\begin{array}{l}\text { Mostly because of cardiovascular reasons but my legs were also } \\
\text { hurting and the bar felt heavy on my shoulders. }\end{array}$ & & & & $\mathbf{x}$ & $\mathbf{x}$ \\
\hline $83 \%$ & A general lousy feeling. I just wanted to get it over with. & & & $\mathbf{x}$ & & \\
\hline $83 \%$ & $\begin{array}{l}\text { A general feeling of fatigue of the whole system rather than just } \\
\text { the muscles in my legs. }\end{array}$ & & $\mathbf{x}$ & & & \\
\hline $83 \%$ & $\begin{array}{l}\text { I run out of power and knew I wouldn't be able to complete } \\
\text { another repetition. }\end{array}$ & & $\mathbf{x}$ & & & \\
\hline \multicolumn{7}{|c|}{ Bench-press } \\
\hline $70 \%$ & The limiting factor was fatigue in my left shoulder muscles. & $\mathbf{x}$ & & & & \\
\hline $70 \% *$ & I never felt my chest muscles fatigued like this before. & $\mathbf{x}$ & & & & \\
\hline $70 \% *$ & I can't really explain it, I felt that I just ran out of strength. & & $\mathbf{x}$ & & & \\
\hline $70 \%$ & My heartrate. I felt it from the very first repetition. & & & & $\mathbf{x}$ & \\
\hline $83 \% *$ & $\begin{array}{l}\text { Without a doubt it was my chest muscles. Not my triceps as I } \\
\text { expected. }\end{array}$ & $\mathbf{x}$ & & & & \\
\hline $83 \%$ & $\begin{array}{l}\text { The limiting factor was mostly psychological. As if I gave up on } \\
\text { the next repetition. }\end{array}$ & & & $\mathbf{x}$ & & \\
\hline $83 \%$ & Pain in my left wrist. & & & & & $\mathbf{x}$ \\
\hline $83 \%$ & I ran out of strength in my chest muscles. & $\mathbf{x}$ & & & & \\
\hline $83 \% *$ & $\begin{array}{l}\text { A combination of different reasons, including lower back pain, } \\
\text { fatigue in my shoulder and arm muscles, and a feeling of } \\
\text { breathlessness. }\end{array}$ & $\mathbf{x}$ & & & $\mathbf{x}$ & $\mathbf{x}$ \\
\hline $83 \%$ & $\begin{array}{l}\text { The limiting factor was my ability to produce strength with my } \\
\text { upper body. }\end{array}$ & $\mathbf{x}$ & & & & \\
\hline
\end{tabular}

1 


\section{Table 3 (on next page)}

Table 3

Mixed model logistic regression results. OR - odds ratio; SE - standard error; $\mathrm{Cl}$ - confidence interval; LL - lower limit; UL - upper limit. 


\begin{tabular}{|c|c|c|c|c|c|c|c|c|}
\hline Model & $\begin{array}{c}\text { Random } \\
\text { terms }\end{array}$ & Fixed terms & OR & $\begin{array}{c}\text { Estimate } \\
(b)\end{array}$ & SE & $\mathbf{Z}$ & $\begin{array}{c}p \text { - } \\
\text { value }\end{array}$ & $\begin{array}{l}95 \% \text { CI } \\
\text { (LL, UL) }\end{array}$ \\
\hline $\begin{array}{l}\operatorname{Pain}_{i j} \sim \operatorname{logistic}\left(b_{0 i j}+\right. \\
b_{1} * \text { Squat_Exercise } e_{i j}+\end{array}$ & \multirow[t]{2}{*}{$\begin{array}{l}b_{0 j}=\gamma_{00}+u_{0 j} \\
b_{1}=\gamma_{10}+u_{1 j}\end{array}$} & 83\%1RM_Condition ${ }_{i j}$ & 0.16 & -1.81 & 0.93 & -1.92 & 0.053 & $-12.43,0.01$ \\
\hline $\begin{array}{c}b_{2} * 83 \% 1 R M_{-} \text {Condition }_{i j+}+ \\
\left.u_{0 j}\right)\end{array}$ & & Squat_Exercise $_{i j}$ & 4.74 & 1.55 & 3.59 & 0.43 & 0.665 & $-6.87,7.38$ \\
\hline $\begin{array}{c}\text { Negative_affect } t_{i j} \sim \text { logistic } \\
\left(b_{0 i j}+\right.\end{array}$ & \multirow[t]{2}{*}{$b_{0 j}=\gamma_{00}+u_{0 j}$} & 83\%1RM_Condition ${ }_{i j}$ & 0.85 & -0.16 & 0.55 & -0.29 & 0.770 & $-1.44,1.05$ \\
\hline $\begin{array}{c}\left(b_{0 i j}+\right. \\
b_{1} * \text { Squat_Exercise }_{i j}+ \\
b_{2}{ }^{* 83 \% 1 R M_{-} \text {Condition }_{i j}+} \\
\left.u_{0 j}\right)\end{array}$ & & Squat_Exercise $_{i j}$ & 4.59 & 1.52 & 3.59 & 2.27 & 0.022 & $0.50,16.11$ \\
\hline $\begin{array}{c}\text { General_fatigue }_{i j} \sim \\
\operatorname{logistic}\left(b_{0 i j}+\right.\end{array}$ & \multirow[t]{2}{*}{$b_{0} j=\gamma_{00}+u_{0 j}$} & 83\%1RM_Condition ${ }_{i j}$ & 0.99 & -0.01 & 0.38 & -0.04 & 0.967 & $-0.85,0.81$ \\
\hline $\begin{array}{c}\text { logistic }\left(b_{0 i j}+\right. \\
b_{1} * \text { Squat_Exercise }_{i j}+ \\
b_{2} * 83 \% 1 R M_{-} \text {Condition }_{i j+}+ \\
\left.u_{0 j}\right)\end{array}$ & & Squat_Exercise $_{i j}$ & 1.06 & 0.06 & 0.38 & 0.17 & 0.860 & $-0.71,0.92$ \\
\hline $\begin{array}{c}\text { Muscle_fatigue }_{i j} \sim \text { logistic } \\
\left(b_{0 i j}+\right.\end{array}$ & \multirow[t]{2}{*}{$b_{0 j}=\gamma_{00}+u_{0 j}$} & 83\%1RM_Condition ${ }_{i j}$ & 1.84 & 0.61 & 0.35 & 1.72 & 0.084 & $-0.05,1.38$ \\
\hline $\begin{array}{c}\left(b_{0 i j}+\right. \\
b_{1} * \text { Squat_Exercise }_{i j}+ \\
b_{2} * 83 \% 1 \text { EM_Condition }_{i j+}+ \\
\left.u_{0 j}\right)\end{array}$ & & Squat_Exercise $_{i j}$ & 0.63 & -0.45 & 0.35 & -1.30 & 0.192 & $-1.19,0.26$ \\
\hline \multirow[b]{2}{*}{$\begin{array}{c}\left(b_{0 i j}+\right. \\
b_{1} * \text { Squat_Exercise }_{i j}+ \\
b_{2} * 83 \% 1 R M_{-} \text {Condition }_{j} \\
\left.u_{0 j}\right)\end{array}$} & \multirow[t]{2}{*}{$b_{0 j}=\gamma_{00}+u_{0 j}$} & 83\%1RM_Condition ${ }_{i j}$ & 0.23 & -1.44 & 0.75 & -1.91 & 0.056 & $\begin{array}{c}-10.80,- \\
0.001 \\
\end{array}$ \\
\hline & & Squat_Exercise $_{i j}$ & 3.29 & 1.19 & 0.70 & 1.69 & 0.090 & $-0.11,5.03$ \\
\hline
\end{tabular}


\begin{tabular}{|l|l|l|l|l|l|l|}
\hline & & & & & \\
\hline
\end{tabular} 


\section{Table 4 (on next page)}

\section{Table 4}

Frequency (percent) of each category by gender and set endpoint condition. 
Table 4. Frequency (percent) of each category by gender and set endpoint condition.

\begin{tabular}{|c|c|c|c|c|c|c|}
\hline \multicolumn{2}{|c|}{} & $\begin{array}{c}\text { Muscle } \\
\text { fatigue }\end{array}$ & $\begin{array}{c}\text { General } \\
\text { fatigue }\end{array}$ & $\begin{array}{c}\text { Negative } \\
\text { affect }\end{array}$ & Pain & Cardio \\
\hline Gender & Females & $42 / 80$ & $19 / 80$ & $7 / 80$ & $10 / 80$ & $12 / 80$ \\
& & $(52.5 \%)$ & $(23.8 \%)$ & $(8.7 \%)$ & $(12.5 \%)$ & $(15 \%)$ \\
& Males & $42 / 76$ & $22 / 76$ & $8 / 76$ & $8 / 76$ & $6 / 76$ \\
$(55.3 \%)$ & $(28.9 \%)$ & $(10.5 \%)$ & $(10.5 \%)$ & $(7.9 \%)$ \\
\hline Set & MF & $25 / 37$ & $8 / 37$ & $0 / 37$ & $4 / 37$ & $2 / 37$ \\
endpoint & & $(67.5 \%)$ & $(21.6 \%)$ & $(0 \%)$ & $(10.8 \%)$ & $(5.4 \%)$ \\
& RM & $60 / 121$ & $33 / 121$ & $16 / 121$ & $15 / 121$ & $16 / 121$ \\
& & $(49.6 \%)$ & $(27.3 \%)$ & $(13.2 \%)$ & $(12.4 \%)$ & $(13.2 \%)$ \\
\hline
\end{tabular}

1 